\title{
ON A NEW CLASS OF FUNCTIONAL SPACES WITH APPLICATION TO THE VELOCITY AVERAGING
}

\author{
Martin Lazar and Darko Mitrović
}

University of Dubrovnik, Croatia and University of Montenegro, Montenegro

\begin{abstract}
We introduce a new family of functional spaces which incorporate Bochner spaces $\mathrm{L}^{p}\left(\mathbf{R}^{m} ; E\right)$, with $E$ being an appropriate Banach space, and to which we extend the H-distributions. We use the developed theory to prove a general version of the velocity averaging lemma in a heterogeneous $\mathrm{L}^{p}, p \leq 2$ setting.
\end{abstract}

\section{INTRODUCTION AND MOTIVATION}

In [7] the authors prove the velocity averaging result for a sequence of solutions to problems determined by a general (pseudo)differential equation operator:

$$
\mathcal{P} u_{n}(\mathbf{x}, \mathbf{y})=\sum_{k=1} \partial_{x_{k}}^{\alpha_{k}}\left(a_{k}(\mathbf{x}, \mathbf{y}) u_{n}(\mathbf{x}, \mathbf{y})\right)=\partial_{\mathbf{y}}^{\kappa} G_{n}(\mathbf{x}, \mathbf{y}),
$$

where $(\mathbf{x}, \mathbf{y}) \in \mathbf{R}^{d+m}, \alpha_{k}>0$ are real numbers, $\partial_{x_{k}}^{\alpha_{k}}$ are (the Fourier) multiplier operators with the symbols $\left(2 \pi i \xi_{k}\right)^{\alpha_{k}}$, while $\partial_{\mathbf{p}}^{\kappa}=\partial_{p_{1}}^{\kappa_{1}} \ldots \partial_{p_{m}}^{\kappa_{m}}$ for a multi-index $\boldsymbol{\kappa}=\left(\kappa_{1}, \ldots, \kappa_{m}\right) \in \mathbf{N}^{m}$.

More precisely, assuming that $u_{n} \rightarrow 0$ in $\mathrm{L}^{p}\left(\mathbf{R}^{d+m}\right)$, for $p \in\langle 1,2]$, and that the principal symbol $A$ of the operator $\mathcal{P}$ satisfies the restrictive nondegeneracy condition:

$$
\text { (a.e. } \left.(\mathbf{x}, \mathbf{y}) \in \mathbf{R}^{d+m}\right), \quad A(\mathbf{x}, \mathbf{y}, \boldsymbol{\xi}):=\sum_{k \in I^{\prime}} a_{k}(\mathbf{x}, \mathbf{y})(2 \pi i \boldsymbol{\xi})^{\boldsymbol{\alpha}_{k}} \neq 0 \quad(\forall \boldsymbol{\xi} \in \mathrm{P})
$$

2010 Mathematics Subject Classification. 35A27, 46B50, 47G30.

Key words and phrases. H-distributions, velocity averaging, non-degeneracy condition. 
we were able to prove that the sequence of averaged quantities

$$
\left(\int_{\mathbf{R}^{m}} \rho(\mathbf{y}) u_{n}(\mathbf{x}, \mathbf{y}) d \mathbf{y}\right)
$$

converges to 0 strongly in $\mathrm{L}_{\text {loc }}^{1}\left(\mathbf{R}^{d}\right)$ for any $\rho \in \mathrm{C}_{\mathrm{c}}\left(\mathbf{R}^{m}\right)$.

Here the dual variable $\boldsymbol{\xi}$ takes values in a suitably defined manifold $\mathrm{P}$ determined by the order of derivatives from (1.1) (see Remark 1.3 for details).

The main tool in the proof of the above result are $\mathrm{H}$-distributions $[1,7]$ - a generalisation of the concept of H-measures $[2,12]$ to $\mathrm{L}^{p}, p \leq 2$ setting, whose the most up-to-date version we provide below.

TheOREM 1.1. Let $\left(u_{n}\right)$ be a bounded sequence in $\mathrm{L}^{p}\left(\mathbf{R}^{d+m}\right), p \in\langle 1,2]$, and let $\left(v_{n}\right)$ be a sequence converging weakly to zero in $\mathrm{L}^{2}\left(\mathbf{R}^{d}\right) \cap \mathrm{L}^{q}\left(\mathbf{R}^{d}\right)$ for some (finite) $q \geq p^{\prime}$. Let $\bar{p} \in[1, p\rangle$ be such that $\frac{1}{p}+\frac{1}{\bar{p}^{\prime}}+\frac{1}{q}=1$. Then, after passing to a subsequence (not relabeled), there exists a continuous bilinear functional $B$ on $\mathrm{L}^{p^{\prime}}\left(\mathbf{R}^{m} ; \mathrm{L}^{\bar{p}^{\prime}}\left(\mathbf{R}^{d}\right)\right) \otimes \mathrm{C}^{d}(\mathrm{P})$ (with $\mathrm{L}^{\infty}\left(\mathbf{R}^{d}\right)$ being replaced by $\mathrm{C}_{0}\left(\mathbf{R}^{d}\right)$ if $\left.q=p^{\prime}\right)$ such that for every $\phi_{1} \in \mathrm{L}^{p^{\prime}}\left(\mathbf{R}^{m} ; \mathrm{L}^{\bar{p}^{\prime}}\left(\mathbf{R}^{d}\right)\right), \phi_{2} \in \mathrm{C}_{0}\left(\mathbf{R}^{d}\right)$, and $\psi \in \mathrm{C}^{d}(\mathrm{P})$, it holds

$$
B\left(\phi_{1} \overline{\phi_{2}}, \bar{\psi}\right)=\lim _{n \rightarrow \infty} \int_{\mathbf{R}^{d+m}} \phi_{1}(\mathbf{x}, \mathbf{y}) u_{n}(\mathbf{x}, \mathbf{y}) \overline{\mathcal{A}_{\psi_{\mathrm{P}}}\left(\phi_{2} v_{n}\right)(\mathbf{x})} d \mathbf{x} d \mathbf{y}
$$

where $\mathcal{A}_{\psi_{\mathrm{P}}}$ is the (Fourier) multiplier operator on $\mathbf{R}^{d}$ associated to $\psi \circ \pi_{\mathrm{P}}$ and $\pi_{\mathrm{P}}$ is a projection on the manifold $\mathrm{P}$ given by (1.5) below.

The functional B we call the H-distribution corresponding to (sub)sequences (of) $\left(u_{n}\right)$ and $\left(v_{n}\right)$.

The proof of the theorem can be found in the Appendix.

Like H-measures, H-distributions provide an information on a (potential) strong convergence of the corresponding sequence(s). In particular, if the functional $B$ associated to sequences of solutions $u_{n}$ and

$$
v_{n}=\frac{\int_{\mathbf{R}^{m}} \rho(\mathbf{y}) u_{n}(\mathbf{x}, \mathbf{y}) d \mathbf{y}}{\left|\int_{\mathbf{R}^{m}} \rho(\mathbf{y}) u_{n}(\mathbf{x}, \mathbf{y}) d \mathbf{y}\right|}
$$

is zero (with $\rho \in \mathrm{C}_{\mathrm{c}}\left(\mathbf{R}^{m}\right)$ being arbitrary), then for the product we have

$$
u_{n} v_{n}=\left|\int_{\mathbf{R}^{m}} \rho(\mathbf{y}) u_{n}(\mathbf{x}, \mathbf{y}) d \mathbf{y}\right| \longrightarrow 0 \quad \text { in } \quad \mathrm{L}_{\mathrm{loc}}^{1}\left(\mathbf{R}^{d}\right)
$$

which is exactly the corresponding velocity averaging result.

Thus the velocity averaging result follows directly once showing that corresponding H-distribution is trivial. Crucial in that direction is the following result [7, Corollary 2.3].

Corollary 1.2. If $\bar{p}>1$ the bilinear functional $B$ defined in Theorem 1.1 can be extended as a continuous functional on $\mathrm{L}^{p^{\prime}}\left(\mathbf{R}^{m} ; \mathrm{L}^{\bar{p}^{\prime}}\left(\mathbf{R}^{d} ; \mathrm{C}^{d}(\mathrm{P})\right)\right)$. 

tained

By standard arguments, starting from the equation (1.1) in [7] we ob-

$$
A B=0 .
$$

Taking coefficients $a_{k} \in \mathrm{L}^{\bar{p}^{\prime}}\left(\mathbf{R}^{d+m}\right)$ and assuming the non-degeneracy condition (1.2) one gets $\chi_{A=0}=0$ in $\mathrm{L}^{p^{\prime}}\left(\mathbf{R}^{m} ; \mathrm{L}^{\bar{p}^{\prime}}\left(\mathbf{R}^{d} ; \mathrm{C}^{d}(\mathrm{P})\right)\right)$. By Corollary 1.2 the distribution $B$ is continuous on that space, which implies that $B=0$, thus providing the result.

The same idea, of extending an appropriate functional determined by a sequence of solutions of (1.1) to a Banach space in which the characteristic function of the principal symbol equals one, was used in the $\mathrm{L}^{2}$ framework with the aim of (generalised) H-measures [5, 6, 9].

The general result on an extension of a bilinear functional on $\mathrm{L}^{p}\left(\mathbf{R}^{d}\right) \otimes E$, where $\mathrm{E}$ is a separable Banach space, can be found in [7].

The motivation for the current paper was to weaken the non-degeneracy assumption (1.2), and to obtain an analogue result under the standard nondegeneracy condition (e.g. $[3,10,11])$, i.e. to replace $(1.2)$ by

$$
\text { (a.e. } \left.\left.\mathbf{x} \in \mathbf{R}^{d}\right),(\forall \boldsymbol{\xi} \in \mathrm{P}) \quad A(\mathbf{x}, \mathbf{y}, \boldsymbol{\xi}) \neq 0 \quad \text { (a.e. } \mathbf{y} \in \mathbf{R}^{m}\right) \text {. }
$$

Such a generalisation would allow one to consider a larger family of (pseudo)differential operators. As a standard example, one can consider the operator with the principal symbol $p(y, \boldsymbol{\xi})=\xi_{1}+y \xi_{2}$ obviously satisfying (1.4), but not obeying its stronger counterpart (1.2).

It is easy to check that condition (1.4) implies $\chi_{A=0}=0$ in the space $\mathrm{L}^{\bar{p}^{\prime}}\left(\mathbf{R}^{d} ; \mathrm{C}^{d}\left(\mathrm{P} ; \mathrm{L}^{p^{\prime}}\left(\mathbf{R}^{m}\right)\right)\right)$. Thus, following the above ideas one would first try to extend $\mathrm{H}$-distributions as continuous functionals on the mentioned space. However, such an extension turned out to be beyond the capacity of techniques familiar to the authors. For that reason we take another approach based on a class of suitable defined functional spaces presented in the next section. Section 3 provides the extension result for the $\mathrm{H}$-distributions, while in the subsequent one we apply the developed theory to the velocity averaging problem. The paper is closed by the conclusion section pointing toward some open problems and feasible generalisations of the obtained results. The existence result for $\mathrm{H}$-distributions, which are the basic tool used in the paper, is presented in the Appendix.

REMARK 1.3. We assume that the order of derivatives $\alpha_{k j}$ entering the principal symbol are either integers, or larger or equal to the space dimension $d$ (thus providing appropriate smoothness of the principal symbol with respect to the dual variable).

The manifold $\mathrm{P}$ is determined by the relation

$$
\mathrm{P}=\left\{\boldsymbol{\xi} \in \mathbf{R}^{d}: \sum_{i=1}^{d}\left|\xi_{i}\right|^{l \alpha_{i}}=1\right\},
$$


where $l$ is a minimal number such that either $l \alpha_{i}>d$ or $l \alpha_{i}$ is an even integer for each $i$. These assumptions ensure that the introduced manifold is of class $\mathrm{C}^{d}$ which enables one to analyse associated multipliers, as well as to define appropriate variant of the H-distributions on them (see Theorem 1.1).

In order to associate an $\mathrm{L}^{p}$ multiplier to a function defined on $\mathrm{P}$ we extend it to $\mathbf{R}^{d} \backslash\{0\}$ by means of the projection

$$
\left(\pi_{\mathrm{P}}(\boldsymbol{\xi})\right)_{i}=\xi_{i}\left(\left|\xi_{1}\right|^{l \alpha_{1}}+\cdots+\left|\xi_{d}\right|^{l \alpha_{d}}\right)^{-1 / l \alpha_{i}}=\xi_{i}|\boldsymbol{\xi}|_{\boldsymbol{\alpha}}^{-1 / \alpha_{i}}, \quad i=1, \ldots, d,
$$

for all $\boldsymbol{\xi} \in \mathbf{R}^{d} \backslash\{0\}$, where here and in the sequel we use abbreviation $|\boldsymbol{\xi}|_{\boldsymbol{\alpha}}=$ $\left(\sum\left|\xi_{i}\right|^{l \alpha_{i}}\right)^{1 / l}$.

There are many criteria on a symbol $\psi$ providing it to be an $\mathrm{L}^{p}$ multiplier. A standard one is the Marcinkiewicz multiplier theorem [4, Theorem 5.2.4], where one can find rather precise $\mathrm{L}^{p}$-bound of the functional $\mathcal{A}_{\psi}$ with respect to $\psi$. Throughout the paper $\mathcal{A}_{\psi}$ stands for a Fourier multiplier operator defined by

$$
\mathcal{A}_{\psi}(u)=\overline{\mathcal{F}}(\psi \hat{u}),
$$

where $\hat{u}(\boldsymbol{\xi})=\mathcal{F}(u)(\boldsymbol{\xi})=\int_{\mathbf{R}^{d}} e^{-2 \pi i \mathbf{x} \cdot \boldsymbol{\xi}} u(x) d x$ is the Fourier transform while $\overline{\mathcal{F}}$ (or $\vee$ ) is the inverse Fourier transform.

The Marcinkiewicz multiplier theorem requires a symbol being of class $\mathrm{C}^{d}$ (at least outside coordinate hyperplanes), which is the main reason for which we have to consider smooth functions with respect to the dual variable $\boldsymbol{\xi}$. In [6, Lemma 5] we have proven that for any $\psi \in \mathrm{C}^{d}(\mathrm{P})$, the composition $\psi \circ \pi$ is an $\mathrm{L}^{p}$ multiplier satisfying

$$
\left\|\mathcal{A}_{\psi_{\mathrm{P}}}\right\|_{\mathrm{L}^{p} \rightarrow \mathrm{L}^{p}} \leq C\|\psi\|_{\mathrm{C}^{d}(\mathrm{P})},
$$

with a constant $C$ depending only on $p \in\langle 1, \infty\rangle$ and $d$. Here and in the sequel, for $\psi \in \mathrm{C}^{d}(\mathrm{P})$ we shall denote by $\mathcal{A}_{\psi_{\mathrm{P}}}$ the multiplier operator corresponding to the extension $\psi \circ \pi_{\mathrm{P}}$.

\section{W SPACES}

For $s \in\langle 1, \infty\rangle$ and $\bar{s}\rangle s$, let $r$ be defined as $1 / s^{\prime}+1 / \bar{s}=1 / r$. We introduce a functional $\|\cdot\|_{W^{(\bar{s}, s)}}$ by the relation

$$
\|\phi\|_{W^{(\bar{s}, s)}}=\sup _{\|\rho\|_{s^{\prime}=1}}\left(\int_{\mathbf{R}^{d}}\left\|\int_{\mathbf{R}^{m}} \rho(\mathbf{x}, \mathbf{y}) \phi(\mathbf{x}, \mathbf{y}, \boldsymbol{\xi}) d \mathbf{y}\right\|_{\mathrm{C}^{d}(\mathrm{P})}^{r} d \mathbf{x}\right)^{1 / r}
$$

for any function $\phi$ for which the right hand side makes sense. Here, $\|\rho\|_{s^{\prime}}=$ $\|\rho\|_{L^{s^{\prime}}\left(\mathbf{R}^{d+m}\right)}$.

It is easily checked that

$$
W^{(\bar{s}, s)}\left(\mathbf{R}^{d}, \mathbf{R}^{m}, \mathrm{P}\right)=W^{(\bar{s}, s)}:=\left\{\phi:\|\phi\|_{W^{(\bar{s}, s)}}<\infty\right\}
$$

defines a vector space. 
REMARK 2.1. Roughly speaking, an element from the introduced $W^{(\bar{s}, s)}$ space is an $L^{s}$ function with respect to $\mathbf{y}$, while an $\mathrm{L}^{\bar{s}}$ function with respect to $\mathbf{x}$. Obviously, it contains tensor products of the form $\varphi \otimes \psi \epsilon$ $\mathrm{L}^{\bar{s}}\left(\mathbf{R}^{d} ; \mathrm{L}^{s}\left(\mathbf{R}^{m}\right)\right) \otimes \mathrm{C}^{d}(\mathrm{P})$.

By identifying two functions $\phi_{1}, \phi_{2} \in W^{(\bar{s}, s)}$ such that

$$
\text { (a.e. } \left.\mathbf{x} \in \mathbf{R}^{d}\right)\left(\forall \boldsymbol{\xi} \in \text { P) (a.e. } \mathbf{y} \in \mathbf{R}^{m}\right) \quad \phi_{1}-\phi_{2}=0
$$

relation (2.1) defines a norm on $W^{(\bar{s}, s)}$.

A function $\phi$ is zero in $W^{(\bar{s}, s)}$ if and only if

$$
\text { (a.e. } \left.\mathbf{x} \in \mathbf{R}^{d}\right),(\forall \boldsymbol{\xi} \in \mathrm{P}) \quad \phi(\mathbf{x}, \mathbf{y}, \boldsymbol{\xi})=0 \quad\left(\text { a.e. } \mathbf{y} \in \mathbf{R}^{m}\right) \text {. }
$$

REMARK 2.2. Notice also that it is easy to check that the space $\mathrm{L}^{\bar{s}}\left(\mathbf{R}^{d} ; \mathrm{L}^{s}\left(\mathbf{R}^{m} ; \mathrm{C}^{d}(\mathrm{P})\right)\right)$ is continuously embedded into $W^{(\bar{s}, s)} ;$ just note that

$$
\left\|\int_{\mathbf{R}^{m}} \rho(\mathbf{x}, \mathbf{y}) \phi(\mathbf{x}, \mathbf{y}, \boldsymbol{\xi}) d \mathbf{y}\right\|_{\mathrm{C}^{d}(\mathrm{P})} \leq \int_{\mathbf{R}^{m}} \mid \rho(\mathbf{x}, \mathbf{y})\|\| \phi(\mathbf{x}, \mathbf{y}, \cdot) \|_{\mathrm{C}^{d}(\mathrm{P})} d \mathbf{y} .
$$

Let us finally analyse mutual relations between introduced spaces for different values of multi-indices $(\bar{s}, s)$. In particular we would like to check if $W^{(\bar{q}, q)} \hookrightarrow W^{(\bar{s}, s)}$ for $(\bar{q}, q)>(\bar{s}, s)$. As it is the case with standard Lebesgue spaces, corresponding embedding is obtained for localised versions $W^{(\bar{s}, s)}\left(\Omega_{x}, \Omega_{y}, \mathrm{P}\right)$, where $\Omega_{x}$ and $\Omega_{y}$ are relatively compact sets in $\mathbf{R}^{d}$ and $\mathbf{R}^{m}$, respectively. These spaces are defined in the same manner as the above ones, just by restricting test functions $\rho$ in $(2.1)$ to $\mathrm{L}^{s^{\prime}}\left(\Omega_{x} \times \Omega_{y}\right)$. The result is given by the next lemma.

Lemma 2.3. Let $q>s$ and $1 / s^{\prime}+1 / \bar{s}=1 / q^{\prime}+1 / \bar{q}=1 / r$. Then the following continuous embedding holds

$$
W^{(\bar{q}, q)}\left(\Omega_{x}, \Omega_{y}, \mathrm{P}\right) \hookrightarrow W^{(\bar{s}, s)}\left(\Omega_{x}, \Omega_{y}, \mathrm{P}\right) .
$$

Proof. First note that the assumptions directly imply $\bar{q}>\bar{s}$.

In order to prove the result, recall that for any $1<q^{\prime}<s^{\prime}$, it holds

$$
\|\rho\|_{\mathrm{L}^{q^{\prime}}\left(\Omega_{x} \times \Omega_{y}\right)} \leq C\|\rho\|_{\mathrm{L}^{s^{\prime}}\left(\Omega_{x} \times \Omega_{y}\right)}
$$

for a constant $C$ depending on $\Omega_{x} \times \Omega_{y}, s^{\prime}$ and $q^{\prime}$. Thus

$$
\begin{aligned}
\|\phi\|_{W^{(s, \bar{s})}} & =\sup _{\|\rho\|_{s^{\prime}}=1}\left(\int_{\mathbf{R}^{d}}\left\|\int_{\mathbf{R}^{m}} \rho(\mathbf{x}, \mathbf{y}) \phi(\mathbf{x}, \mathbf{y}, \boldsymbol{\xi}) d \mathbf{y}\right\|_{\mathrm{C}^{d}(\mathrm{P})}^{r} d \mathbf{x}\right)^{1 / r} \\
& \leq \sup _{\|\rho\|_{q^{\prime}} \leq C}\left(\int_{\mathbf{R}^{d}}\left\|\int_{\mathbf{R}^{m}} \rho(\mathbf{x}, \mathbf{y}) \phi(\mathbf{x}, \mathbf{y}, \boldsymbol{\xi}) d \mathbf{y}\right\|_{\mathrm{C}^{d}(\mathrm{P})}^{r} d \mathbf{x}\right)^{1 / r} \\
& =C\|\phi\|_{W^{(q, \bar{q})}},
\end{aligned}
$$

which implies (2.2). 
Now, we can provide a precise definition of the space to which $\mathrm{H}$ distributions are extended.

For each $N \in \mathbf{N}$, we partition the spaces $\mathbf{R}^{d}$ and $\mathbf{R}^{m}$ on disjoint hypercubes with the edge parallel to the coordinate axis and of the length $1 / 2^{N}$ (let us call it the $N$-partition). We assume that a vertex of some hypercube is at the origin, so that the $N+1$-partition is simply obtained by partitioning each of the cubes from the $N$-partition on another $2^{d}$ equal size cubes.

Let $\Upsilon$ be a family of functions of the form

$$
\Upsilon=\left\{\sum_{i, j=1}^{\tilde{N}} \alpha_{i j}(\boldsymbol{\xi}) \chi_{i}^{N}(\mathbf{x}) \chi_{j}^{N}(\mathbf{y}): \alpha_{i, j}^{N} \in \mathrm{C}^{d}(\mathrm{P}), N, \tilde{N} \in \mathbf{N}\right\},
$$

where $\chi_{i}^{N}$ and $\chi_{j}^{N}$ are characteristic functions of appropriate hypercubes from the $N$-partitions of $\mathbf{R}^{d}$ and $\mathbf{R}^{m}$. By $\tilde{W}^{(\bar{s}, s)}$ we denote the closure of $\Upsilon$ in $W^{(\bar{s}, s)}$.

As step functions, like in ordinary $\mathrm{L}^{p}$ spaces, are dense in Bochner spaces (cf. [15]), the family $\Upsilon$ is dense in the space $\mathrm{L}^{\bar{s}}\left(\mathbf{R}^{d} ; \mathrm{L}^{s}\left(\mathbf{R}^{m} ; \mathrm{C}^{d}(\mathrm{P})\right)\right)$. As by Remark 2.2 the latter one is continuously embedded into $W^{(\bar{s}, s)}$, it follows that $\tilde{W}^{(\bar{s}, s)}$ contains that space as well. As a consequence of that embedding, a velocity averaged result relied on the extension of H-distributions to $\tilde{W}^{(\bar{s}, s)}$, will incorporate results obtained under restrictive non-degeneracy condition (1.2) proved in [8] (see the final section).

\section{Extension of H-Distributions to $\tilde{W}$}

First, we need the following commutation lemma.

LEMMA 3.1. Let $\left(u_{n}\right)$ be a bounded, uniformly compactly supported sequence in $\mathrm{L}^{\infty}\left(\mathbf{R}^{d}\right)$, converging to 0 in the sense of distributions. Then for any $b \in \mathrm{L}^{s}\left(\mathbf{R}^{d}\right), s>1$ arbitrary, and any symbol $\psi \in \mathrm{C}^{d}(\mathrm{P})$ it holds

$$
\lim _{n \rightarrow \infty}\left\|b \mathcal{A}_{\psi}\left(u_{n}\right)-\mathcal{A}_{\psi}\left(b u_{n}\right)\right\|_{\mathrm{L}^{r}\left(\mathbf{R}^{d}\right)}=0, \quad r \in\langle 1, s\rangle .
$$

Proof. The difference between the current lemma and the Tartar commutation lemma [13, Lemma 28.2] is in the regularity of the function $b$. Namely, in the cited one it is assumed that $b \in \mathrm{C}\left(\mathbf{R}^{d}\right)$ and it is restricted to $\mathrm{L}^{2}$ setting. However, since we have better assumptions on the sequence $\left(u_{n}\right)$ (in our case, it belongs to $\mathrm{L}^{p}\left(\mathbf{R}^{d}\right)$ for every $p \geq 1$ ), we can easily prove (3.1).

Indeed, let $\left(b_{\varepsilon}\right)$ be a family of smooth functions such that $\left\|b_{\varepsilon}-b\right\|_{L^{s}\left(\mathbf{R}^{d}\right)} \rightarrow$ 0 as $\varepsilon \rightarrow 0$. It holds

$$
\begin{aligned}
\left\|b \mathcal{A}_{\psi}\left(u_{n}\right)-\mathcal{A}_{\psi}\left(b u_{n}\right)\right\|_{\mathrm{L}^{r}\left(\mathbf{R}^{d}\right)} \leq & \left\|b \mathcal{A}_{\psi}\left(u_{n}\right)-b_{\varepsilon} \mathcal{A}_{\psi}\left(u_{n}\right)\right\|_{\mathrm{L}^{r}\left(\mathbf{R}^{d}\right)} \\
& +\left\|b_{\varepsilon} \mathcal{A}_{\psi}\left(u_{n}\right)-\mathcal{A}_{\psi}\left(b_{\varepsilon} u_{n}\right)\right\|_{\mathrm{L}^{r}\left(\mathbf{R}^{d}\right)} \\
& +\left\|\mathcal{A}_{\psi}\left(b_{\varepsilon} u_{n}\right)-\mathcal{A}_{\psi}\left(b u_{n}\right)\right\|_{\mathrm{L}^{r}\left(\mathbf{R}^{d}\right)} .
\end{aligned}
$$


In $[6$, Theorem 6$]$ it was proven that projection $\pi_{\mathrm{P}}$ used throughout the paper (note that $\mathcal{A}_{\psi}$ stands for $\mathcal{A}_{\psi \circ \pi_{\mathrm{P}}}$ ) satisfies conditions of the Tartar commutation lemma. Thus the lemma, together with the interpolation inequality implies the middle term on the right hand side converges to zero with respect to $n$. Estimating other two terms by the Hölder inequality and the Marcinkiewcz theorem we get

$$
\lim _{n \rightarrow \infty}\left\|b \mathcal{A}_{\psi}\left(u_{n}\right)-\mathcal{A}_{\psi}\left(b u_{n}\right)\right\|_{\mathrm{L}^{r}\left(\mathbf{R}^{d}\right)} \leq C\left\|b_{\varepsilon}-b\right\|_{\mathrm{L}^{s}\left(\mathbf{R}^{d}\right)} .
$$

Letting $\varepsilon \rightarrow 0$ here, we conclude the lemma.

Now, we can prove the following theorem.

THEOREM 3.2. In addition to the assumptions of Theorem 1.1 we assume that the sequence $\left(v_{n}\right)$ is bounded in $\mathrm{L}^{\infty}\left(\mathbf{R}^{d}\right)$ and uniformly supported within the compact set $K \subset \mathbf{R}^{d}$. Then the bilinear functional $B: \mathrm{L}^{\bar{p}^{\prime}}\left(\mathbf{R}^{d} ; \mathrm{L}^{p^{\prime}}\left(\mathbf{R}^{m}\right)\right) \times$ $\mathrm{C}^{d}(\mathrm{P})$ defined by $(1.3)$ can be extended as a continuous linear functional on the space $\tilde{W}^{\left(\bar{p}^{\prime}, p^{\prime}\right)}$.

Proof. According to the definition of the space $\tilde{W}^{\left(\bar{p}^{\prime}, p^{\prime}\right)}$, it is enough to prove that we can extend the functional $B$ on $\Upsilon$ endowed with the topology determined by the norm (2.1).

Thus, we need to prove that for $\phi=\sum_{i, j=1}^{\tilde{N}} \alpha_{i j}(\boldsymbol{\xi}) \chi_{i}^{N}(\mathbf{x}) \chi_{j}^{N}(\mathbf{y})$

$$
|\langle B, \phi\rangle| \leq C\|\phi\|_{W^{\left(\bar{p}^{\prime}, p^{\prime}\right)}},
$$

for a constant $C$ independent of $\phi$.

According to the definition of the functional $B$ and (the commutation) Lemma 3.1, we have

$$
\langle B, \phi\rangle=\lim _{n \rightarrow \infty} \int_{\mathbf{R}^{d+m}} \sum_{i, j=1}^{\tilde{N}} u_{n}(\mathbf{x}, \mathbf{y}) \chi_{i}^{N}(\mathbf{x}) \chi_{j}^{N}(\mathbf{y}) \mathcal{A}_{\alpha_{i j}}\left(\chi_{i}^{N} v_{n}\right)(\mathbf{x}) d \mathbf{x} d \mathbf{y} .
$$

Note that due to the uniform compactly support assumption on the sequence $\left(v_{n}\right)$ the above sum with respect to $i$ is taken just for indices $i$ for which the corresponding hypercube intersects the set $K$.

By applying the Hölder inequality, we get

$$
\begin{aligned}
|\langle B, \phi\rangle| \leq \limsup _{n} & \int_{\mathbf{R}^{d+m}}\left(\sum_{i, j=1}^{\tilde{N}}\left|u_{n}(\mathbf{x}, \mathbf{y})\right|^{p} \chi_{i}^{N}(\mathbf{x}) \chi_{j}^{N}(\mathbf{y})\right)^{1 / p} \times \\
& \times\left(\sum_{i, j=1}^{\tilde{N}}\left|\mathcal{A}_{\alpha_{i j}}\left(\chi_{i}^{N} v_{n}\right)(\mathbf{x})\right|^{p^{\prime}} \chi_{i}^{N}(\mathbf{x}) \chi_{j}^{N}(\mathbf{y})\right)^{1 / p^{\prime}} d \mathbf{x} d \mathbf{y}
\end{aligned}
$$




$$
\begin{aligned}
& \leq \underset{n}{\limsup }\left\|\sum_{i, j=1}^{\tilde{N}} \chi_{i}^{N} \chi_{j}^{N} u_{n}\right\|_{L^{p}\left(\mathbf{R}^{d+m}\right)}\left\|\sum_{i, j=1}^{\tilde{N}} \mathcal{A}_{\alpha_{i j}}\left(\chi_{i}^{N} v_{n}\right) \chi_{i}^{N}(\mathbf{x}) \chi_{j}^{N}(\mathbf{y})\right\|_{\mathrm{L}^{p^{\prime}}\left(\mathbf{R}^{d+m}\right)} \\
& \leq \underset{n}{\limsup }\left\|u_{n}\right\|_{\mathrm{L}^{p}\left(\mathbf{R}^{d+m}\right)}\left\|\sum_{i, j=1}^{\tilde{N}} \mathcal{A}_{\alpha_{i j}}\left(\chi_{i}^{N} v_{n}\right) \chi_{i}^{N}(\mathbf{x}) \chi_{j}^{N}(\mathbf{y})\right\|_{\mathrm{L}^{p^{\prime}}\left(\mathbf{R}^{d+m}\right)} .
\end{aligned}
$$

Let us now estimate the $\mathrm{L}^{p^{\prime}}$-norm of the function

$$
\sum_{i, j=1}^{\tilde{N}} \mathcal{A}_{\alpha_{i j}}\left(\chi_{i}^{N} v_{n}\right)(\mathbf{x}) \chi_{i}^{N}(\mathbf{x}) \chi_{j}^{N}(\mathbf{y})
$$

It defines a linear functional on $\mathrm{L}^{p}\left(\mathbf{R}^{d+m}\right)$ via:

$$
\text { (3.3) } \mathrm{L}^{p}\left(\mathbf{R}^{d+m}\right) \ni \rho \mapsto \int_{\mathbf{R}^{d+m}} \rho(\mathbf{x}, \mathbf{y}) \sum_{i, j=1}^{\tilde{N}} \mathcal{A}_{\alpha_{i j}}\left(\chi_{i}^{N} v_{n}\right)(\mathbf{x}) \chi_{i}^{N}(\mathbf{x}) \chi_{j}^{N}(\mathbf{y}) d \mathbf{x} d \mathbf{y}
$$

whose norm is equal to the norm of the corresponding function. Before we start estimating its norm, let us notice that for almost every $\mathbf{x} \in \mathbf{R}^{d}$ and every $a \in \mathrm{L}_{l o c}^{1}\left(\mathbf{R}^{m}\right)$ :

$$
\int_{\mathbf{R}^{m}} a(\mathbf{y}) \sum_{j} \mathcal{A}_{\alpha_{i j}}\left(\chi_{i}^{N} v_{n}\right)(\mathbf{x}) \chi_{j}^{N}(\mathbf{y}) d \mathbf{y}=\mathcal{A}_{\int_{\mathbf{R}^{m}} a(\mathbf{y}) \sum_{j} \alpha_{i j} \chi_{j}^{N} d \mathbf{y}}\left(\chi_{i}^{N} v_{n}\right)(\mathbf{x}) .
$$

Indeed, it is enough to recall that

$$
\begin{aligned}
\int_{\mathbf{R}^{m}} \sum_{j} a_{j}(\mathbf{y}) \mathcal{A}_{\psi_{j}(\xi)} v(\mathbf{x}) d \mathbf{y} & =\int_{\mathbf{R}^{m}} \sum_{j} a_{j}(\mathbf{y})\left(\psi_{j}(\xi) \hat{v}\right)^{\vee}(\mathbf{x}) d \mathbf{y} \\
& =\left(\int_{\mathbf{R}^{m}} \sum_{j} a_{j}(\mathbf{y}) \psi_{j}(\xi) d \mathbf{y} \hat{v}\right)^{\vee}(\mathbf{x}) \\
& :=\mathcal{A}_{\int_{\mathbf{R}^{m}} \sum_{j} a_{j}(\mathbf{y}) \psi_{j}(\xi) d \mathbf{y}} v(\mathbf{x}) .
\end{aligned}
$$

Now, we can estimate the norm of the functional defined by

$$
\sum_{i, j=1}^{\tilde{N}} \mathcal{A}_{\alpha_{i j}}\left(\chi_{i}^{N} v_{n}\right)(\mathbf{x}) \chi_{i}^{N}(\mathbf{x}) \chi_{j}^{N}(\mathbf{y}) .
$$

According to the density of the step functions, it is enough to take

$$
\rho(\mathbf{x}, \mathbf{y})=\sum_{i=1}^{\tilde{N}} \chi_{i}^{N}(\mathbf{x}) \rho_{i}(\mathbf{y}) \in \mathrm{L}^{p}\left(\mathbf{R}^{d+m}\right)
$$

in (3.3). Remark that we can take same $N$ here as in (3.2) since we can refine the sum either here or in (3.2) without loosing the form of the corresponding 
functions. For such chosen $\rho$, it holds:

$$
\begin{aligned}
& \left|\int_{\mathbf{R}^{d+m}} \sum_{i, j=1}^{\tilde{N}} \rho_{i}(\mathbf{y}) \chi_{i}^{N}(\mathbf{x}) \mathcal{A}_{\alpha_{i j}}\left(\chi_{i}^{N} v_{n}\right)(\mathbf{x}) \chi_{j}^{N}(\mathbf{y}) d \mathbf{x} d \mathbf{y}\right| \\
& \leq \int_{\mathbf{R}^{d}}\left(\sum_{i=1}^{\tilde{N}} \chi_{i}^{N} \chi_{K}\right)^{1 / q}\left(\sum_{i}\left|\mathcal{A} \int_{\mathbf{R}^{m} \sum_{j=1}^{\tilde{N}} \rho_{i} \alpha_{i j} \chi_{j}^{N} d \mathbf{y}}\left(\chi_{i}^{N} v_{n}\right)\right|^{q^{\prime}}\right)^{1 / q^{\prime}} d \mathbf{x},
\end{aligned}
$$

where $\frac{1}{q^{\prime}}=\frac{1}{p}+\frac{1}{\bar{p}^{\prime}}$. Denote by $C_{v}$ the $\mathrm{L}^{\infty}$-bound of the sequence $\left(v_{n}\right)$. Using the Marcinkiewicz theorem and Hölder inequality again, we obtain

$$
\begin{aligned}
& \left|\int_{\mathbf{R}^{d+m}} \rho(\mathbf{x}, \mathbf{y}) \sum_{i, j=1}^{\tilde{N}} \mathcal{A}_{\alpha_{i j}}\left(\chi_{i}^{N} v_{n}\right)(\mathbf{x}) \chi_{j}^{N}(\mathbf{y}) d \mathbf{x} d \mathbf{y}\right| \\
& \leq C \operatorname{meas}(K)^{1 / q}\left(\int_{\mathbf{R}^{d}} \sum_{i=1}^{\tilde{N}}\left\|\int_{\mathbf{R}^{m}} \sum_{j=1}^{\tilde{N}} \rho_{i}(\mathbf{y}) \alpha_{i j}(\boldsymbol{\xi}) \chi_{j}^{N}(\mathbf{y}) d \mathbf{y}\right\|_{\mathrm{C}^{d}(\mathrm{P})}^{q^{\prime}} \chi_{i}^{N}\left|v_{n}\right|^{q^{\prime}} d \mathbf{x}\right)^{1 / q^{\prime}} \\
& \leq C_{v, K}\left(\int_{\mathbf{R}^{d}}\left\|\int_{\mathbf{R}^{m}} \sum_{i, j=1}^{\tilde{N}} \rho_{i}(\mathbf{y}) \alpha_{i j}(\boldsymbol{\xi}) \chi_{j}^{N}(\mathbf{y}) \chi_{i}^{N}(\mathbf{x}) d \mathbf{y}\right\|_{\mathrm{C}^{d}(\mathrm{P})}^{q^{\prime}} d \mathbf{x}\right)^{1 / q^{\prime}} \\
& \leq C_{v, K}\|\rho\|_{\mathrm{L}^{p}}\left\|\sum_{i, j=1}^{\tilde{N}} \alpha_{i j}(\boldsymbol{\xi}) \chi_{j}^{N}(\mathbf{y}) \chi_{i}^{N}(\mathbf{x})\right\|_{W}
\end{aligned}
$$

where $C_{v, K}$ is a constant depending on $\|v\|_{\mathrm{L}^{\infty}}$ and $K$. This proves the theorem.

\section{Application to the velocity averaging}

By means of the analytical results obtained in the previous sections we prove a velocity averaging results for a sequence of problems (1.1). Let us first recall a corollary of the Marcinkiewicz multiplier theorem [4, Theorem 5.2.4].

Corollary 4.1. Suppose that $\psi \in \mathrm{C}^{d}\left(\mathbf{R}^{d} \backslash \cup_{j=1}^{d}\left\{\xi_{j}=0\right\}\right)$ is a bounded function such that for some constant $C>0$ it holds

$$
\left|\boldsymbol{\xi}^{\tilde{\alpha}} \partial^{\tilde{\boldsymbol{\alpha}}} \psi(\boldsymbol{\xi})\right| \leq C, \quad \boldsymbol{\xi} \in \mathbf{R}^{d} \backslash \cup_{j=1}^{d}\left\{\xi_{j}=0\right\}
$$

for every multi-index $\tilde{\boldsymbol{\alpha}}=\left(\tilde{\alpha}_{1}, \ldots, \tilde{\alpha}_{d}\right) \in \mathbf{N}_{0}^{d}$ such that $|\tilde{\boldsymbol{\alpha}}|=\tilde{\alpha}_{1}+\tilde{\alpha}_{2}+\cdots+$ $\tilde{\alpha}_{d} \leq d$. Then, the function $\psi$ is an $\mathrm{L}^{p}$-multiplier for $p \in\langle 1, \infty\rangle$, and the operator norm of $\mathcal{A}_{\psi}$ depends on $C, p$ and d only.

Relying on this result, we proved the following lemma in [8], whose proof we repeat for the sake of completeness. 
Lemma 4.2. Let $\boldsymbol{\alpha} \in \mathbf{R}_{+}^{d}$ and let $\theta: \mathbf{R}^{d} \rightarrow \mathbf{R}$ be a smooth compactly supported function equal to one on the unit ball centred at origin.

Then for any $\gamma>0$ the multiplier operator $\mathcal{T}^{\gamma}$ with the symbol

$$
T^{\gamma}(\boldsymbol{\xi})(1-\theta(\boldsymbol{\xi}))=\frac{1}{|\boldsymbol{\xi}|_{\boldsymbol{\alpha}}^{\gamma}}(1-\theta(\boldsymbol{\xi}))
$$

is a continuous $\mathrm{L}^{p}\left(\mathbf{R}^{d}\right) \rightarrow \mathrm{W}^{\gamma \boldsymbol{\alpha}, p}\left(\mathbf{R}^{d}\right)$ operator for any $p \in\langle 1, \infty\rangle$. Specially, due to the Rellich theorem it is a compact $\mathrm{L}^{p}\left(\mathbf{R}^{d}\right) \rightarrow \mathrm{L}_{\mathrm{loc}}^{p}\left(\mathbf{R}^{d}\right)$ operator.

REMARK 4.3. Here and in the sequel by $\mathrm{W}^{\boldsymbol{\alpha}, q}\left(\mathbf{R}^{d}\right)$ we denote the anisotropic space defined as

$$
\mathrm{W}^{\boldsymbol{\alpha}, q}\left(\mathbf{R}^{d}\right):=\left\{u \in \mathrm{L}^{q}\left(\mathbf{R}^{d}\right): \partial_{k}^{\alpha_{k}} u \in \mathrm{L}^{q}\left(\mathbf{R}^{d}\right), k=1, \ldots, d\right\},
$$

whose dual is denoted by $\mathrm{W}^{-\boldsymbol{\alpha}, q^{\prime}}\left(\mathbf{R}^{d}\right)$ (for details on anisotropic Sobolev spaces see e.g. [14]).

Proof. We shall first prove that the operator $\mathcal{T}^{\gamma}$ is a continuous operator on $\mathrm{L}^{p}\left(\mathbf{R}^{d}\right)$. To this effect, remark that it is enough to prove that $T^{\gamma}$ satisfies condition of Corollary 4.1 away from the origin. Around the origin, the operator $\mathcal{T}^{\gamma}$ is controlled by the term $(1-\theta)$ (which is equal to zero on $B(0,1)$ and obviously satisfies conditions of Corollary 4.1$)$. We use the induction argument with respect to the order of derivative in (4.1).

(i) $n=1$

In this case, we compute

$$
\partial_{k} T^{\gamma}(\boldsymbol{\xi})=C_{k} \frac{1}{\xi_{k}} T^{\gamma}(\boldsymbol{\xi})\left(\pi_{\mathrm{P}}(\boldsymbol{\xi})\right)_{k}^{l \alpha_{k}}
$$

for some constant $C_{k}$. From here, it obviously follows $\left|\xi_{k} \partial_{k} T^{\gamma}(\boldsymbol{\xi})\right| \leq C$ for $\boldsymbol{\xi} \in \mathbf{R}^{d}$ away from the origin.

(ii) $n=m$

Our inductive hypothesis is that a $\boldsymbol{\alpha}$-order derivatives of $T^{\gamma}(\boldsymbol{\xi})$ can be represented in the following way

$$
\partial^{\boldsymbol{\alpha}} T^{\gamma}(\boldsymbol{\xi})=\frac{1}{\boldsymbol{\xi}^{\boldsymbol{\alpha}}} T^{\gamma}(\boldsymbol{\xi}) P_{\boldsymbol{\alpha}}(\boldsymbol{\xi})
$$

where $P_{\boldsymbol{\alpha}}$ is a bounded function satisfying (4.1) for $|\tilde{\boldsymbol{\alpha}}| \leq d-|\boldsymbol{\alpha}|$.

(iii) $n=m+1$

To prove that (4.2) holds for $|\boldsymbol{\alpha}|=m+1$ it is enough to notice that $\boldsymbol{\alpha}=\mathrm{e}_{k}+\boldsymbol{\alpha}^{\prime}$, where $\left|\boldsymbol{\alpha}^{\prime}\right|=m$, and that according to the induction hypothesis we have

$$
\partial^{\boldsymbol{\alpha}} T^{\gamma}=\partial_{k} \partial^{\boldsymbol{\alpha}^{\prime}} T^{\gamma}=\partial_{k}\left(\frac{1}{\boldsymbol{\xi}^{\boldsymbol{\alpha}^{\prime}}} T^{\gamma}(\boldsymbol{\xi}) P_{\boldsymbol{\alpha}^{\prime}}(\boldsymbol{\xi})\right)=\frac{1}{\boldsymbol{\xi}^{\boldsymbol{\alpha}}} T^{\gamma}(\boldsymbol{\xi}) P_{\boldsymbol{\alpha}}(\boldsymbol{\xi}),
$$

where

$$
P_{\boldsymbol{\alpha}}(\boldsymbol{\xi})=\left(P_{\mathrm{e}_{k}} P_{\boldsymbol{\alpha}^{\prime}}+\xi_{k} \partial_{k} P_{\boldsymbol{\alpha}^{\prime}}-\alpha_{k} P_{\boldsymbol{\alpha}^{\prime}}\right)(\boldsymbol{\xi}),
$$


thus satisfying conditions (4.1) as well.

From here, (4.1) immediately follows for $T^{\gamma}$ away from the origin, thus proving that the operator $\mathcal{T}^{\gamma}$ is a continuous operator on $\mathrm{L}^{p}\left(\mathbf{R}^{d}\right)$.

It remains to prove that for any $\alpha_{j}$ from the $d$-tuple $\boldsymbol{\alpha}=\left(\alpha_{1}, \ldots, \alpha_{d}\right)$, the multiplier operator $\partial_{x_{j}}^{\gamma \alpha_{j}} \mathcal{T}^{\gamma}$ is a continuous $\mathrm{L}^{p}\left(\mathbf{R}^{d}\right) \rightarrow \mathrm{L}^{p}\left(\mathbf{R}^{d}\right)$ operator. To accomplish this, notice that its symbol is

$$
(1-\theta(\boldsymbol{\xi})) \frac{\left(2 \pi i \xi_{j}\right)^{\gamma \alpha_{j}}}{|\boldsymbol{\xi}|_{\boldsymbol{\alpha}}^{\gamma}}=(1-\theta(\boldsymbol{\xi}))\left(\pi_{\mathrm{P}}(\boldsymbol{\xi})\right)_{j}^{\gamma \alpha_{j}} .
$$

Thus, away from the origin, it is a composition of a function which is smooth outside coordinate hyperplanes and the projection $\pi_{\mathrm{P}}$, and by Lemma A.1 satisfies continuity relation (1.6).

The following theorem holds.

THEOREM 4.4. For a sequence of equations (1.1) assume the following:

- $u_{n} \rightarrow 0$ in $\mathrm{L}^{p}\left(\mathbf{R}^{d+m}\right)$, for $p \in\langle 1,2]$.

- For some $\bar{p} \in\langle 1, p\rangle$, it holds

$$
a_{k} \in \mathrm{L}^{\bar{p}^{\prime}}\left(\mathbf{R}^{d} ; \mathrm{L}^{p^{\prime}}\left(\mathbf{R}^{m}\right)\right), \quad k \in I, \quad \frac{1}{p}+\frac{1}{\bar{p}^{\prime}}+\frac{1}{q}=\frac{1}{p}+\frac{1}{p^{\prime}}=1,
$$

where $q=\frac{p \bar{p}}{p-\bar{p}}$, while $p^{\prime}$ stands for a dual index of $p$.

- The sequence $\left(G_{n}\right)$ is strongly precompact in the anisotropic space $\mathrm{L}^{1}\left(\mathbf{R}^{m} ; \mathrm{W}^{-\boldsymbol{\alpha}, q^{\prime}}\left(\mathbf{R}^{d}\right)\right)$.

- the principal symbol $A$ satisfies the following strong convergence

$$
\left(\frac{|A|^{2}}{|A|^{2}+\delta}\right)_{\mid K} \longrightarrow \chi_{K} \quad \text { in } \quad W^{\left(\bar{p}^{\prime}, p^{\prime}\right)}
$$

as $\delta \longrightarrow 0$ for every compact $K$.

Then, for any $\rho \in \mathrm{C}_{\mathrm{c}}\left(\mathbf{R}^{m}\right)$, the sequence of averaged quantities

$$
\left(\int_{\mathbf{R}^{m}} \rho(\mathbf{y}) u_{n}(\mathbf{x}, \mathbf{y}) d \mathbf{y}\right)
$$

converges to 0 strongly in $\mathrm{L}_{\text {loc }}^{1}\left(\mathbf{R}^{d}\right)$.

Proof. The proof essentially follows the line of the proof of $[6$, Theorem 7 , which provides an analogous result in $\mathrm{L}^{2}$ setting. Here we present the basic steps adapted to the framework of this paper.

For fixed $\rho \in \mathrm{C}_{\mathrm{c}}\left(\mathbf{R}^{m}\right)$ and $\varphi \in \mathrm{C}_{\mathrm{c}}\left(\mathbf{R}^{d}\right)$, denote by $V$ a weak-* $\mathrm{L}^{\infty}\left(\mathbf{R}^{d}\right)$ limit along some subsequence (not relabelled) of the sequence of functions

$$
V_{n}= \begin{cases}\frac{\varphi(\mathbf{x}) \int_{\mathbf{R}^{m}} \rho(\mathbf{y}) u_{n}(\mathbf{x}, \mathbf{y}) d \mathbf{y}}{\left|\int_{\mathbf{R}^{m}} \rho(\mathbf{y}) u_{n}(\mathbf{x}, \mathbf{y}) d \mathbf{y}\right|}, & \int_{\mathbf{R}^{m}} \rho(\mathbf{y}) u_{n}(\mathbf{x}, \mathbf{y}) d \mathbf{y} \neq 0 \\ 0, & \text { otherwise. }\end{cases}
$$


Denote $v_{n}=V_{n}-V$ and remark that $v_{n} \stackrel{*}{\rightarrow} 0$ in $\mathrm{L}^{\infty}\left(\mathbf{R}^{d}\right)$.

Take a dual product of (1.1) with the test function of the form

$$
\rho_{1}(\mathbf{y})\left(\mathcal{T} \circ \mathcal{A}_{\psi_{\mathrm{P}}}\right)\left(\varphi_{1} v_{n}\right)(\mathbf{x})
$$

where $\psi, \varphi_{1}$ and $\rho_{1}$ are arbitrary smooth test functions in variables $\boldsymbol{\xi}, \mathbf{x}$ and $\mathbf{y}$, respectively, while $\mathcal{T}$ is a multiplier operator with the symbol essentially equal to $|\boldsymbol{\xi}|_{\boldsymbol{\alpha}}^{-1}$. According to Lemma $4.2, \mathcal{T}$ is a continuous operator $\mathrm{L}^{s}\left(\mathbf{R}^{d}\right) \rightarrow$ $\mathrm{W}^{\boldsymbol{\alpha}, s}\left(\mathbf{R}^{d}\right)$ for any $s \in\langle 1, \infty\rangle$.

Letting $n \rightarrow \infty$, taking into account the strong convergence of $\left(G_{n}\right)$, and the relation (1.3) one gets

$$
A B=0,
$$

where $B$ is an $H$-distribution determined by the sequences $\left(\rho u_{n}\right)$ and $\left(v_{n}\right)$. Due to their uniform support (with respect to $\mathbf{y}$ and $\mathbf{x}$ variable), the support of $B$ is contained within the set $K=\operatorname{supp} \varphi \times \operatorname{supp} \rho$.

By means of the extension of H-distributions provided by Theorem 3.2, we can take a test function in (4.5) of the form

$$
\frac{\phi(\mathbf{x}, \mathbf{y}) \psi(\boldsymbol{\xi}) \overline{A(\mathbf{x}, \mathbf{y}, \boldsymbol{\xi})}}{|A(\mathbf{x}, \mathbf{y}, \boldsymbol{\xi})|^{2}+\delta}
$$

for some arbitrary $\delta>0$ and $\phi \otimes \psi \in \mathrm{C}_{\mathrm{c}}\left(\mathbf{R}^{d+m}\right) \otimes \mathrm{C}^{d}(\mathrm{P})$. By passing to the limit as $\delta \rightarrow 0$ and using the convergence assumption (4.4), one gets $B=0$.

In order to finish the proof, for the previously chosen $\rho$ and $\varphi$ take in (1.3) test functions of the form $\psi=1$ and $\phi_{1}(\mathbf{x}, \mathbf{y})=\varphi(\mathbf{x}) \rho_{2}(\mathbf{y})$, where $\rho_{2} \in \mathrm{C}_{\mathrm{c}}\left(\mathbf{R}^{m}\right)$ equals to one on the support of $\rho$. Since $B=0$, from the definition of the sequence $\left(v_{n}\right)$ (keep also in mind that $u_{n} \rightarrow 0$ in $\mathrm{L}^{s}\left(\mathbf{R}^{d+m}\right)$ ), it follows

$$
\lim _{n \rightarrow \infty} \int_{\mathbf{R}^{d}}|\varphi(\mathbf{x})|^{2}\left|\int_{\mathbf{R}^{m}} \rho(\mathbf{y}) u_{n}(\mathbf{x}, \mathbf{y}) d \mathbf{y}\right| d \mathbf{x}=0
$$

which concludes the proof (due to arbitrariness of $\rho$ and $\varphi$ ).

\section{Conclusion}

In order to simplify the presentation, the results and theory within this paper have been restricted to (pseudo)differential equations of the form (1.1), but are easily extended to more generalised ones, containing terms with mixed derivatives:

$$
\mathcal{P} u_{n}(\mathbf{x}, \mathbf{y})=\sum_{k \in I} \partial_{\mathbf{x}}^{\boldsymbol{\alpha}_{k}}\left(a_{k}(\mathbf{x}, \mathbf{y}) u_{n}(\mathbf{x}, \mathbf{y})\right)=\partial_{\mathbf{y}}^{\kappa} G_{n}(\mathbf{x}, \mathbf{y}) .
$$

Here $I$ stands for a finite set of indices, and $\partial_{\mathbf{x}}^{\boldsymbol{\alpha}_{k}}=\partial_{x_{1}}^{\alpha_{k 1}} \ldots \partial_{x_{d}}^{\alpha_{k d}}$ for a multiindex $\boldsymbol{\alpha}_{k}=\left(\alpha_{k 1}, \ldots, \alpha_{k d}\right) \in\left(\mathbf{R}_{0}^{+}\right)^{d}$. The principal symbol $A$ of the operator 
$\mathcal{P}$ in that case is defined by

$$
A(\mathbf{x}, \mathbf{y}, \boldsymbol{\xi}):=\sum_{k \in I^{\prime}} a_{k}(\mathbf{x}, \mathbf{y})(2 \pi i \boldsymbol{\xi})^{\boldsymbol{\alpha}_{k}}
$$

where the sum is taken over all terms from (5.1) whose order of derivative $\boldsymbol{\alpha}_{k}$ is not dominated by any other multi-index from $I$.

The coefficients $a_{k}$ entering equations (1.1) are assumed to belong to the space $\mathrm{L}^{\bar{p}^{\prime}}\left(\mathbf{R}^{d} ; \mathrm{L}^{p^{\prime}}\left(\mathbf{R}^{m}\right)\right)$ for some $\bar{p} \in\langle 1, p\rangle$, i.e. they are almost taken from the dual of the space $\mathrm{L}^{p}\left(\mathbf{R}^{d+m}\right)$ containing the solutions. The reason for excluding the limit, dual index $p^{\prime}$ with respect to $\mathbf{x}$ variable lies in the commutation lemma A.2 (in Appendix), which provides compactness of the commutator only in $\mathrm{L}^{q}, q \in\langle 1, \infty\rangle$ (limit cases $q=1$ and $q=\infty$ are excluded). The cited commutation lemma is applied in the space $\mathbf{R}^{d}$ containing the state variable $\mathbf{x}$, but not the velocity variable $\mathbf{y}$.

In [7] a velocity averaging result similar to the one obtained in previous section has been obtained with coefficients satisfying the restrictive nondegeneracy condition (1.2). As this condition implies (actually it is equivalent to) the following strong convergence

$$
\left(\frac{|A|^{2}}{|A|^{2}+\delta}\right)_{\mid K} \longrightarrow \chi_{K} \quad \text { in } \quad \mathrm{L}^{\bar{p}^{\prime}}\left(\mathbf{R}^{d} ; \mathrm{L}^{p^{\prime}}\left(\mathbf{R}^{m} ; \mathrm{C}^{d}(\mathrm{P})\right)\right)
$$

as $\delta \longrightarrow 0$ (cf. [8]), and the last space is continuously embedded into $W^{\left(\bar{p}^{\prime}, p^{\prime}\right)}$ (see the end of Section 2), the result of Theorem 4.4 generalises the one obtained in [7].

The presented velocity averaging result is obtained under convergency assumption (4.4). What remains as an interesting open question is a possibility of replacing it by a standard non-degeneracy condition (1.4). A method of obtaining an affirmative answer would rely on the localisation principle (4.5) and show that it locally (i.e. on each bounded subdomain) implies

$$
\chi_{A \neq 0} B=0 .
$$

According to the standard non-degeneracy conditions (1.4) and the definition of introduced $W$ spaces, locally it holds

$$
\chi_{A \neq 0} \equiv 1 \text { in } W^{\left(\bar{p}^{\prime}, p^{\prime}\right)},
$$

which together with (5.3) and the derived extension of H-distributions (Theorem 3.2) would result in $B \equiv 0$. This would immediately imply strong $\mathrm{L}_{\text {loc }}^{1}\left(\mathbf{R}^{d}\right)$ convergence of the velocity averages (see the comments after Theorem 1.1). However, problem of proving relation (5.3) is out of our reach and it remains open at the moment. We hope that this paper, and specially the introduced functional spaces, pave the way for the answer, and will have a constructive role in the future investigations of the subject. 


\section{ACKNOWLEDGEMENTS}

The research is supported in part by the Ministry of Science of Montenegro under the project number 01-471, by the Croatian Science Foundation under the project 9780 WeConMApp, by the bilateral Croatian-Montenegro project "Multiscale methods and calculus of variations", and by the FP7 project "Micro-local defect functional and applications" (MiLDeFA) within the frame of the program Marie Curie FP7-PEOPLE-2011-COFUND.

The authors acknowledge the anonymous referee for the careful reading and useful remarks that have improved the final version of the paper.

\section{APPENDIX}

Here, we provide the proof of Theorem 1.1 which lies in the essence of the procedures used in the paper. We need the following result on $\mathrm{L}^{p}$ multipliers arising from the Marcinkiewicz multiplier theorem.

Lemma A.1 ([5, Lemma 5]). For any $\psi \in \mathrm{C}^{d}(\mathrm{P})$, the composition $\psi \circ \pi_{\mathrm{P}}$ is an $\mathrm{L}^{p}$-multiplier, $p \in\langle 1, \infty\rangle$, satisfying bound (1.6).

We also need a generalisation of Tartar's commutation lemma [13, Lemma $28.2]$ to $\mathrm{L}^{p}, p \neq 2$ sequences.

LEMmA A.2. Let $B$ be the operator of multiplication by a continuous function $b \in \mathrm{C}_{0}\left(\mathbf{R}^{d}\right)$. Let $\left(v_{n}\right)$ be a bounded sequence in $\mathrm{L}^{2}\left(\mathbf{R}^{d}\right) \cap \mathrm{L}^{p}\left(\mathbf{R}^{d}\right)$, $p \in[1, \infty]$ such that $v_{n} \rightarrow 0$ in the sense of distributions, and let $\psi \in \mathrm{C}^{d}(\mathrm{P})$. Then for the commutator $C=\mathcal{A}_{\psi_{\mathrm{P}}} B-B \mathcal{A}_{\psi_{\mathrm{P}}}$ the sequence $\left(C v_{n}\right)$ converges strongly to zero in $\mathrm{L}^{q}\left(\mathbf{R}^{d}\right)$ for any $q \in[2, p\rangle$ if $p \geq 2$, and any $q \in\langle p, 2]$ if $p<2$.

PROOF. Remark first that the projection $\psi \circ \pi_{\mathrm{P}}=\psi_{\mathrm{P}}$ satisfies conditions of the variant of the first commutation lemma [13, Lemma 28.2], more precisely, its conditions given below:

$$
\begin{aligned}
& \left(\forall r, \varepsilon \in \mathbf{R}^{+}\right) \quad\left(\exists M \in \mathbf{R}^{+}\right) \\
& \left|\boldsymbol{\eta}_{1}-\boldsymbol{\eta}_{2}\right| \leq r,\left|\boldsymbol{\eta}_{1}\right|,\left|\boldsymbol{\eta}_{2}\right|>M \quad \Longrightarrow \quad\left|\psi\left(\pi_{\mathrm{P}}\left(\boldsymbol{\eta}_{1}\right)\right)-\psi\left(\pi_{\mathrm{P}}\left(\boldsymbol{\eta}_{2}\right)\right)\right| \leq \varepsilon .
\end{aligned}
$$

Indeed, as $\psi$ is an uniformly continuous on $\mathrm{P}$, it is enough to show that for fixed $r$ and $\varepsilon$, the difference $\left|\pi_{\mathrm{P}}\left(\boldsymbol{\eta}_{1}\right)-\pi_{\mathrm{P}}\left(\boldsymbol{\eta}_{2}\right)\right|$ is arbitrary small for $M$ large enough. According to the mean value theorem

$$
\left|\pi_{\mathrm{P}}\left(\boldsymbol{\eta}_{1}\right)-\pi_{\mathrm{P}}\left(\boldsymbol{\eta}_{2}\right)\right| \leq\left|\nabla \pi_{\mathrm{P}}(\boldsymbol{\zeta})\right|\left|\boldsymbol{\eta}_{1}-\boldsymbol{\eta}_{2}\right|,
$$

where $\boldsymbol{\zeta}=\vartheta \boldsymbol{\eta}_{1}+(1-\vartheta) \boldsymbol{\eta}_{2}$ for some $\vartheta \in\langle 0,1\rangle$, and the statement follows as $\nabla \pi_{\mathrm{P}}(\boldsymbol{\eta})$ tends to zero when $|\boldsymbol{\eta}|$ approaches infinity. In other words, conditions of [13, Lemma 28.2] are fulfilled, thus ensuring that $C$ is a compact operator on $\mathrm{L}^{2}\left(\mathbf{R}^{d}\right)$. 
According to the interpolation inequality for any $r$ between 2 and $p$, and $\alpha \in\langle 0,1\rangle$ we have

$$
\left\|C v_{n}\right\|_{q} \leq\left\|C v_{n}\right\|_{2}^{\alpha}\left\|C v_{n}\right\|_{r}^{1-\alpha},
$$

where $1 / q=\alpha / 2+(1-\alpha) / r$. As $C$ is a compact operator on $\mathrm{L}^{2}\left(\mathbf{R}^{d}\right)$, while $C$ is bounded on $\mathrm{L}^{r}\left(\mathbf{R}^{d}\right)$ for $r \in\langle 1, \infty\rangle$, we get the claim.

Finally, we can prove Theorem 1.1.

Proof of TheOrem 1.1. First, remark that according to the commutation lemma (Lemma A.2), it holds

$$
\begin{aligned}
\lim _{n \rightarrow \infty} & \int_{\mathbf{R}^{d+m}} \phi_{1}(\mathbf{x}, \mathbf{y}) u_{n}(\mathbf{x}, \mathbf{y}) \overline{\mathcal{A}_{\psi_{\mathbf{P}}}\left(\phi_{2} v_{n}\right)(\mathbf{x})} d \mathbf{x} d \mathbf{y} \\
& =\lim _{n \rightarrow \infty} \int_{\mathbf{R}^{d+m}} \phi_{1}(\mathbf{x}, \mathbf{y}) \overline{\phi_{2}(\mathbf{x})} u_{n}(\mathbf{x}, \mathbf{y}) \overline{\mathcal{A}_{\psi_{\mathbf{P}}}\left(v_{n}\right)(\mathbf{x})} d \mathbf{x} d \mathbf{y} .
\end{aligned}
$$

Thus the limit in (1.3) depends only on the product $\phi_{1} \overline{\phi_{2}} \in \mathrm{L}^{p^{\prime}}\left(\mathbf{R}^{m} ; \mathrm{L}^{\bar{p}^{\prime}}\left(\mathbf{R}^{d}\right)\right)$.

Next, consider the bilinear mapping $B_{n}$ defined for every $\psi \in \mathrm{C}^{d}(\mathrm{P})$ and $\phi \in \mathrm{L}^{p^{\prime}}\left(\mathbf{R}^{m} ; \mathrm{L}^{\bar{p}^{\prime}}\left(\mathbf{R}^{d}\right)\right)$ by

$$
B_{n}(\phi, \psi)=\int_{\mathbf{R}^{d+m}} \phi(\mathbf{x}, \mathbf{y}) u_{n}(\mathbf{x}, \mathbf{y}) \overline{\mathcal{A}_{\psi}\left(v_{n}\right)(\mathbf{x})} d \mathbf{x} d \mathbf{y} .
$$

According to the Hölder inequality and Lemma A.1, it holds

$$
\left|B_{n}(\phi, \psi)\right| \leq C\|\psi\|_{\mathrm{C}^{d}(\mathrm{P})}\left\|v_{n}\right\|_{\mathrm{L}^{q}\left(\mathbf{R}^{d}\right)} \int_{\mathbf{R}^{m}}\|\phi(\cdot, \mathbf{y})\|_{\mathrm{L}^{\bar{p}^{\prime}}\left(\mathbf{R}^{d}\right)}\left\|u_{n}(\cdot, \mathbf{y})\right\|_{\mathrm{L}^{p}\left(\mathbf{R}^{d}\right)} d \mathbf{y},
$$

where $C$ is the constant from relation (1.6) depending on $d$ and $q$. By using the Hölder inequality again (now applied in the variable $\mathbf{y}$ ), we get that

$$
\begin{aligned}
\left|B_{n}(\phi, \psi)\right| & \leq C\|\psi\|_{\mathrm{C}^{d}(\mathrm{P})}\left\|v_{n}\right\|_{\mathrm{L}^{q}\left(\mathbf{R}^{d}\right)}\|\phi\|_{\mathrm{L}^{p^{\prime}}\left(\mathbf{R}^{m} ; \mathrm{L}^{\bar{p}^{\prime}}\left(\mathbf{R}^{d}\right)\right)}\left\|u_{n}\right\|_{\mathrm{L}^{p}\left(\mathbf{R}^{d+m}\right)} \\
& \leq \bar{C}\|\psi\|_{\mathrm{C}^{d}(\mathrm{P})}\|\phi\|_{\mathrm{L}^{p^{\prime}}\left(\mathbf{R}^{m} ; \mathrm{L}^{\bar{p}^{\prime}}\left(\mathbf{R}^{d}\right)\right)},
\end{aligned}
$$

where $\bar{C}$ depends on $C$, and bounds on $\left\|u_{n}\right\|_{\mathrm{L}^{p}\left(\mathbf{R}^{d+m}\right)}$ and $\left\|v_{n}\right\|_{\mathrm{L}^{q}\left(\mathbf{R}^{d}\right)}$.

Thus it follows that $\left(B_{n}\right)$ is an equibounded sequence of bilinear functionals, and by [1, Lemma 3.2] and (A.3), there exists a functional $B$ for which (1.3) holds.

\section{REFERENCES}

[1] N. Antonić and D. Mitrović, $H$-distributions: an extension of $H$-measures to an $L^{p}-L^{q}$ setting, Abstr. Appl. Anal. 2011, Art. ID 901084, 12 pp.

[2] P. Gérard, Microlocal defect measures, Comm. Partial Differential Equations 16 (1991), 1761-1794.

[3] F. Golse, P.-L. Lions, B. Perthame and R. Sentis, Regularity of the moments of the solution of a transport equation, J. Funct. Anal. 76 (1988), 110-125.

[4] L. Grafakos, Classical Fourier analysis, Springer, New York, 2008.

[5] M. Lazar and D. Mitrović, The velocity averaging for a heterogeneous heat type equation, Math. Commun. 16 (2011), 271-282. 
[6] M. Lazar and D. Mitrović, Velocity averaging - a general framework, Dyn. of Partial Differ. Equ. 9 (2012), 239-260.

[7] M. Lazar and D. Mitrović, On an extension of a bilinear functional on $L^{p}\left(R^{d}\right) \times E$ to a Bochner space with an application to velocity averaging, C. R. Math. Acad. Sci. Paris 351 (2013), 261-264.

[8] M. Lazar and D. Mitrović, On the velocity averaging for equations with optimal heterogeneous rough coefficients, preprint available on arXiv, http://arxiv.org/pdf/1310. 4285.pdf.

[9] E. Yu. Panov, Ultra-parabolic equations with rough coefficients. Entropy solutions and strong precompactness property, J. Math. Sci. (N.Y.) 159 (2009), 180-228.

[10] B. Perthame and P.E. Souganidis, A limiting case for velocity averaging, Ann. Sci. École Norm. Sup. (4) 31 (1998), 591-598.

[11] E. Tadmor and T. Tao, Velocity averaging, kinetic formulations, and regularizing effects in quasi-linear PDEs, Comm. Pure Appl. Math. 60 (2007), 1488-1521.

[12] L. Tartar, $H$-measures, a new approach for studying homogenisation, oscillation and concentration effects in partial differential equations, Proc. Roy. Soc. Edinburgh Sect. A 115 (1990), 193-230.

[13] L. Tartar, The general theory of homogenization: a personalized introduction, Springer-Verlag, Berlin, 2009

[14] L. R. Volevich and B. P. Paneyakh, Certain spaces of generalized functions and embedding theorems, Russ. Math. Surv. 20 (1965), 1-73.

[15] K. Yosida, Functional analysis, Springer-Verlag, Berlin-New York, 1980.

\section{Lazar}

Department of Electrical Engineering and Computing

University of Dubrovnik

Ćira Carića 4, 20000 Dubrovnik

Croatia

E-mail: martin.lazar@unidu.hr

D. Mitrović

Faculty of Mathematics and Natural Sciences

University of Montenegro

Cetinjski put bb, 81000 Podgorica

Montenegro

E-mail: darkom@ac.me

Received: 23.6.2016.

Revised: 11.10.2016. 